\title{
AKARI/AcuA Physical Studies of the Cybele Asteroid Family
}

\author{
KASUGA, Toshihiro \\ (NAOJ)
}

OOTSUBO, Takafumi

(Tohoku University)

\author{
USUI, Fumihiko, HASEGAWA, Sunao \\ (ISAS/JAXA)
}

MÜLLER, Thomas G.

(Max-Planck-Institut für Extraterrestrische Physik)
KURODA, Daisuke

(NAOJ)

ISHIGURO, Masateru

(Seoul National University)
We present a study of 107 Cybele asteroids based on the archival data base "Asteroid Catalog Using AKARI (AcuA)" taken by the infrared astronomical satellite. The data base provides diameters $D>10 \mathrm{~km}$, geometric albedos and taxonomic informations $(75 \%)$ of the Cybeles. We find taxonomic diversity (mainly C-, D- and P-type) in the population of seventy-eight small Cybeles with diameters $10 \mathrm{~km}<D<80 \mathrm{~km}$. Their cumulative power-law size distribution index shows a shallow value of $0.86 \pm 0.03$. By contrast, twenty-nine large Cybeles with $D>80 \mathrm{~km}$ are mostly classified as C- or P- types $(90 \%)$, having a power-law index of $2.39 \pm 0.18$. The total mass of Cybele asteroids is estimated to be $\sim 10^{-5}$ $M_{\text {Earth }}$. We also discuss the origin and formation process of Cybele asteroid family. See [1] for more details.

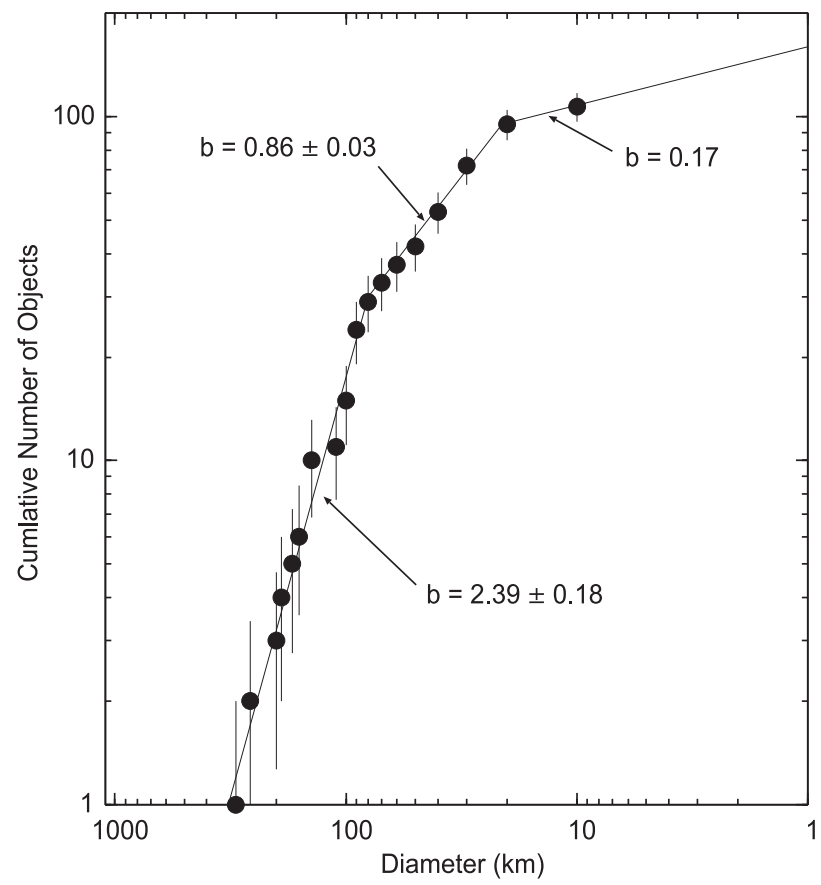

Figure 1: Cumulative size distribution of 107 Cybele asteroids. The derived power-law indexes are $b=0.17(10 \mathrm{~km}<D$ $<20 \mathrm{~km}), 0.86 \pm 0.03(20 \mathrm{~km}<D<80 \mathrm{~km})$ and $2.39 \pm$ $0.18(D>80 \mathrm{~km})$.

Reference

[1] Kasuga, T., et al.: 2012, $A J, \mathbf{1 4 3}, 141$. 